\title{
GAMMA GRAPHS OF SOME SPECIAL CLASSES OF TREES
}

\begin{abstract}
Anna Bień
Abstract. A set $S \subset V$ is a dominating set of a graph $G=(V, E)$ if every vertex $v \in V$ which does not belong to $S$ has a neighbour in $S$. The domination number $\gamma(G)$ of the graph $G$ is the minimum cardinality of a dominating set in $G$. A dominating set $S$ is a $\gamma$-set in $G$ if $|S|=\gamma(G)$.

Some graphs have exponentially many $\gamma$-sets, hence it is worth to ask a question if a $\gamma$-set can be obtained by some transformations from another $\gamma$-set. The study of gamma graphs is an answer to this reconfiguration problem. We give a partial answer to the question which graphs are gamma graphs of trees. In the second section gamma graphs $\gamma \cdot T$ of trees with diameter not greater than five will be presented. It will be shown that hypercubes $Q_{k}$ are among $\gamma . T$ graphs. In the third section $\gamma . T$ graphs of certain trees with three pendant vertices will be analysed. Additionally, some observations on the diameter of gamma graphs will be presented, in response to an open question, published by Fricke et al., if $\operatorname{diam}(T(\gamma))=O(n)$ ?
\end{abstract}

\section{Introduction}

We say that $S \subset V$ is a dominating set of a graph $G=(V, E)$ if every vertex $v \in V$ which does not belong to $S$ has a neighbour in $S$. The domination number $\gamma(G)$ of the graph $G$ is the minimum cardinality of a dominating set in $G$. A dominating set $S$ is a $\gamma$-set in $G$ if $|S|=\gamma(G)$. We use standard notations of graph theory, as in Diestel [1. For a comprehensive introduction to the

Received: 2.05.2015. Revised: 4.06.2015.

(2010) Mathematics Subject Classification: 05C69.

Key words and phrases: dominating set, gamma graph. 
theory of domination in graphs we refer the reader to Haynes, Hedetniemi and Slater [4].

A gamma graph $\gamma \cdot G$ of a graph $G$, defined by Lakshmanan and Vijayakumar [5], is such a graph that $S \subset V(G)$ is a vertex of $\gamma \cdot G$ if $S$ is a $\gamma$-set of G, and $\gamma$-sets $S_{1}$ and $S_{2}$ are adjacent iff there exist two vertices $v, u$ of the graph $G$ such that $S_{1}=S_{2} \backslash\{u\} \cup\{v\}$. Independently, in 2011, four authors initiated the study of a similar class of graphs. The gamma graph $G(\gamma)$ studied by G.H. Fricke, S.M. Hedetniemi, S.T. Hedetniemi and K.R Hutson $[2]$ is a $\gamma . G$ graph, whose vertices fulfill the following condition: if $S_{1}=S_{2} \backslash\{u\} \cup\{v\}$, then $u$ and $v$ are adjacent in the graph $G$. Notice that every graph $G(\gamma)$ is a spanning subgraph of $\gamma . G$, i.e. has the same vertex set. This straightforward observation is worth noting in the context of the research of Lakshmanan and Vijayakumar on the connectivity of a $\gamma \cdot G$ graph. This means that every sufficient condition for $G(\gamma)$ is also valid for $\gamma \cdot G$.

Other class of graphs whose vertices correspond to dominating sets was introduced by Haas and Seyffarth in 2014 [3]. $k$-dominating graph $D_{k}(G)$ is a graph, whose vertices are dominating sets of the graph $G$ such that $|S| \leq k$. Two dominating sets $S_{1}$ and $S_{2}$ are adjacent in $D_{k}(G)$ iff there exist a vertex $v \in V(G)$ such that $S_{1}=S_{2} \cup\{v\}$. These authors also studied the conditions under which such graphs are connected. Their results include the following connection between connectivity of $k$-dominating graphs and gamma graphs: if $D_{\gamma+1}(G)$ is connected, then $\gamma \cdot G$ is connected. Fricke et al. [2] proved that gamma graphs of trees $T(\gamma)$ are connected and asked which graphs are gamma graphs of trees. We will give a partial answer to this question, but with respect to the definition of Lakshmanan Vijayakumar. In the second section gamma graphs $\gamma . T$ of trees with diameter not greater than five will be presented. It will be shown that every gamma graph $\gamma \cdot T$ of such trees is a hypercube $Q_{k}$, a hypercube without one vertex $Q_{k}^{-}$, or a cartesian product $Q_{k} \square Q_{l}^{-}$or $Q_{k}^{-} \square Q_{l}^{-}$. Vertices of $Q_{k}$ correspond to binary sequences of length $k$ and edges correspond to such pairs of sequences which differ in exactly one coordinate. The set of vertices of a cartesian product $G_{1} \square G_{2}$ of two graphs $G_{1}$ and $G_{2}$ is a set $V\left(G_{1}\right) \times V\left(G_{2}\right)$, and $\left(v_{1}, v_{2}\right)$ is adjacent to $\left(u_{1}, u_{2}\right)$ in $G_{1} \square G_{2}$ iff $v_{1}$ is adjacent to $u_{1}$ in $G_{1}, v_{2}=u_{2}$ or $v_{2}$ is adjacent to $u_{2}$ in $G_{2}, v_{1}=u_{1}$. In the third section $\gamma \cdot T$ graphs of certain trees with three pendant vertices will be analysed. Also in response to an open question, if $\operatorname{diam}(T(\gamma))=O(n)$ (see [2]), some observations on the diameter of graphs presented in this paper are noted.

One of the simplest trees are paths. It was shown (see [2]) that $P_{3 k}(\gamma) \simeq$ $K_{1}, P_{3 k+2}(\gamma) \simeq P_{k+2}$ and $P_{3 k+1}(\gamma) \simeq S G(k+1)$, where $S G(k)$ denotes spanning subgraph of a cartesian product of two paths $P_{k} \square P_{k}$ such that

$$
V(S G(k))=\{(i, j): 1 \leq i, j \leq k, i+j \leq k+2\}
$$




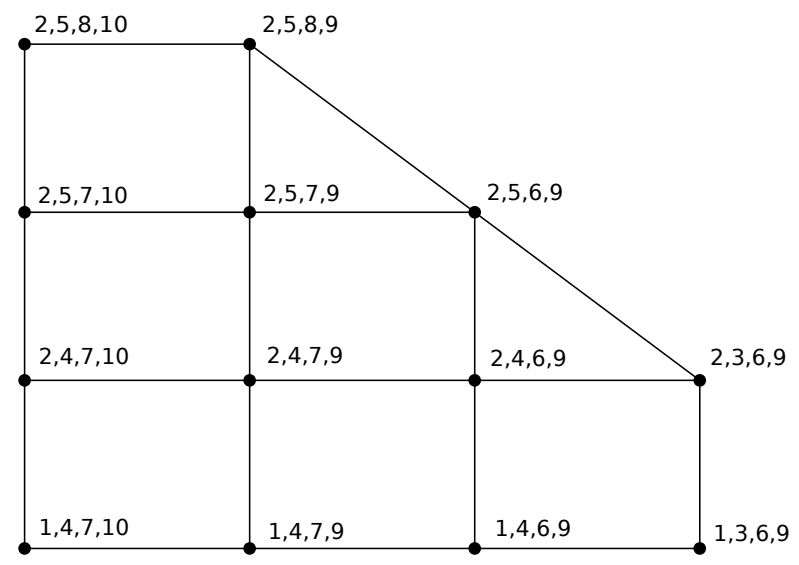

Figure 1. $\gamma \cdot P_{10} \simeq S G^{+}(4)$

and

$$
E(S G(k))=\{((i, j),(k, l)): i=k, l=j+1 ; k=i+1, j=l\}
$$

Notice that $\gamma \cdot P_{3 k} \simeq P_{3 k}(\gamma)$. If $s=3 k+2, k \in \mathbb{N}$, then for every two $\gamma$-sets $S_{1}, S_{2}$ which correspond to vertices of $P_{s}(\gamma)$ : if $S_{1}=S_{2}-\{u\} \cup\{v\}$, then $u$ and $v$ are adjacent in $P_{s}$. Hence $\gamma \cdot P_{3 k+2} \simeq P_{3 k+2}(\gamma) \cdot \gamma \cdot P_{3 k+1}$ (see Figure 1) can be obtained from $P_{3 k+1}(\gamma)$ by adding $k-1$ additional edges. Indeed, if $S=\left\{g_{1}, g_{2}, \ldots, g_{k+1}\right\}$ is a $\gamma$-set of a graph $P_{3 k+1}$ such that $g_{i+1}-g_{i}=1$ for some $i \leq k$, then distances between other consecutive elements of $S$ equal 3 . If $1<i<k$, then $S$ is adjacent in $\gamma \cdot P_{3 k+1}$ to four sets: $S \backslash\left\{g_{i}\right\} \cup\left\{g_{i}-1\right\}$, $S \backslash\left\{g_{i}\right\} \cup\left\{g_{i+1}+1\right\}, S \backslash\left\{g_{i}\right\} \cup\left\{g_{i}-2\right\}, S \backslash\left\{g_{i}\right\} \cup\left\{g_{i}+2\right\}$. If $i=1$ or $i=k$ then $S$ is adjacent to three such sets. $\gamma$-sets of other form have the same degree in both graphs $P_{3 k+1}(\gamma)$ and $\gamma \cdot P_{3 k+1}$. This means that $\gamma \cdot P_{3 k+1} \simeq S G^{+}(k+1)$, where $S G^{+}(k)$ denotes such graph that $V\left(S G^{+}(k)\right)=V(S G(k))$ and

$E\left(S G^{+}(k)\right)=E(S G(k)) \cup\{((i, j)(i+1, j-1)): 2 \leq i \leq k-1, i+j=k+2\}$.

For purpose of this article we define a slide graph. Let $S l(n)$ denote such graph that

$$
V(S l(n))=V\left(S G^{+}(n)\right) \cup\{(n+1,1)\}
$$

and

$$
E(S l(n))=E\left(S G^{+}(n)\right) \cup\{((n, 1)(n+1,1)),((n, 2)(n+1,1))\} .
$$




\section{Gamma graphs of trees with small diameter}

One of the open problems in recent publications on gamma graphs was the question if $\operatorname{diam}(T(\gamma))=O(n)$. We consider a modified version of this problem: is it true that $\operatorname{diam}(\gamma \cdot T)=O(n)$ ? For trees with diameter not greater than five the answer to this question is positive. Let $T$ be a tree, $d=\operatorname{diam}(T)$. For $d \in\{0,1\}$ we have $T \simeq K_{d}, \gamma \cdot T \cong K_{d}$ and $\operatorname{diam}(\gamma \cdot T)=d$. If $d=2$, then $T=K_{1, n}, n>1, \gamma \cdot T \cong K_{1}$ and $\operatorname{diam}(\gamma \cdot T)=1<d$. Since the next lemma can be easily shown by analysing the structure of the graph, we will skip the proof and proceed to more complex cases.

LEMma 2.1. If $T$ is a tree such that $\operatorname{diam}(T)=3$ and $T ¥ P_{4}$, then $\gamma \cdot T \cong K_{1}$ or $\gamma \cdot T \cong K_{2}$.

Lemma 2.2. If $T$ is a tree such that $\operatorname{diam}(T)=4$, then there exist $n \in \mathbb{N}$, such that $\gamma \cdot T \cong Q_{n}$ or $\gamma \cdot T \cong Q_{n}^{-}$.

Proof. Let $S$ be an arbitrary $\gamma$-set of $T$. Note that $T$ contains an induced path $P_{5}$. Let $v_{0}$ be the vertex of this path which is not adjacent to pendant vertices of this path. Note that $d\left(v, v_{0}\right) \leq 2$, for every vertex $v \in V(T)$. Hence $T$ can be obtained from a graph $H$, which is an independent sum of stars $K_{1, n}$ and isolated vertices, by joining a vertex $v_{0}$ to every component of $H$ with one such vertex of this component, which has the maximal degree.

If $H$ has at least two isolated vertices, then $v_{0}$ belongs to $S$, and every vertex not dominated by $v_{0}$ is pendant in $T$ and is dominated by itself or its neighbour. Hence, every element of $S$ different from $v_{0}$ is a vertex of a component of $H$ which is a star $K_{1, n}$. Moreover, $S$ contains exactly one vertex from every star which has a maximal degree in this component. Two $\gamma$-sets $S_{1}$ and $S_{2}$ are adjacent iff $S_{2}=S_{1}-\{v\} \cup\{u\}$, where $u$ and $v$ are two vertices of one component $K_{1,1}$ of $H$. Hence $\gamma \cdot T \simeq Q_{k}$, where $k$ is the number of $K_{1,1}$ s.

If $H$ has one isolated vertex $u$, then $v_{0}$ or $u$ belongs to $S$. Neither $v_{0}$ nor $u$ dominate any vertex $v$ such that $d\left(v_{0}, v\right)=2$. Analogously to the previous case we infer that $S$ contains exactly one vertex of the maximal degree from every component of $H$ which is a star $K_{1, n}$. Also in this case $\gamma \cdot T \simeq Q_{k}$, where $k-1$ is the number of $K_{1,1}$ components of $H$.

If $H$ has no isolated vertices, then $|S|$ is equal to the number of all components of $H$. Note that there is a bijection between the $\gamma$-sets from $V(\gamma \cdot T)$ and binary sequences of length $k$, where $k$ is the number of all $K_{1,1}$ components of $H$. Let enumerate components $K_{1,1}$ of $H$, and define a mapping such that $S \mapsto\left(a_{i}\right)_{i=1}^{k}$, where $a_{i}=1$ whenever $s \in S$ which lies in the $i$-th component is a pendant vertex in $T$, otherwise $a_{i}=0$. If $H$ contains at least one component $K_{1, n}$, where $n>1$, then every $\gamma$-set contains the vertex of this star which 


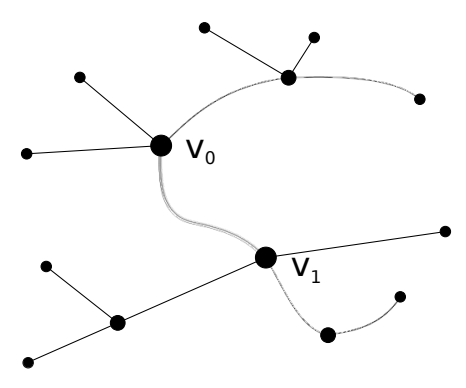

Figure 2. $\operatorname{diam}(T)=5$

dominates $v_{0}$. If $H$ is an independent sum of copies $K_{1,1}$, then the set which contains only pendant vertices and corresponds to the sequence $(1,1,1, \ldots, 1)$ does not dominate $v_{0}$. In this case $\gamma \cdot T \simeq Q_{k}^{-}$.

Since $\operatorname{diam}\left(Q_{k}\right)=k$, we draw the following conclusion from the lemma above.

Corollary 2.3. If $T$ is a tree with $n$ vertices such that $\operatorname{diam}(T)=4$, then $\operatorname{diam}(\gamma \cdot T) \leq n / 2$.

Lemma 2.4. If $T$ is a tree such that $\operatorname{diam}(T)=5$, then there exist $n, m \in \mathbb{N}$ such that $\gamma \cdot T \cong Q_{n}$ or $\gamma \cdot T \cong Q_{n} \square Q_{m}^{-}$or $\gamma \cdot T \cong Q_{n}^{-} \square Q_{m}^{-}$.

Proof. Let $S$ be an arbitrary $\gamma$-set of $T$, and let $v_{0}, v_{1}$ be the vertices of an induced $P_{6}$ subpath of $T$, whose distance from the pendant vertices of the path $P_{6}$ is greater then one (see Figure 2). Let $T_{0}$ and $T_{1}$ denote two components of the graph $T-v_{0} v_{1}$. Assume that $T_{0}$ denotes the component which contains $v_{0}$. Note that $d\left(v, v_{0}\right) \leq 2$ for every vertex $v \in V\left(T_{0}\right)$, and $d\left(v, v_{1}\right) \leq 2$ for every vertex $v \in V\left(T_{1}\right)$. This implies that both graphs $T_{1}$ and $T_{0}$ can be constructed like the tree in the proof of the previous lemma. Let $l$ be the the number of stars $K_{n, 1}$, in the graph $H=T-\left\{v_{0}, v_{1}\right\}$, where $n>1$. Notice that all $l$ vertices whose degree in $H$ is greater than 1 belong to $S$. Moreover, $S$ contains exactly one vertex of every $K_{1,1}$ component of $H$. This means that $|S| \geq l+k$. We enumerate components $K_{1,1}$ of $H$, and consider the mapping $S \mapsto\left(a_{i}^{S}\right)_{i=1}^{k}$ defined in the proof of the previous lemma. If $a^{S}=(0,0, \ldots, 0)$, then $v_{0}$ and $v_{1}$ are dominated by vertices which are not isolated in $H$. It implies that if $H$ does not have isolated vertices, then $\gamma(T)=l+k$.

Let $a^{S i}$ be the subsequence of $a^{S}$ corresponding to the components of $H$ which are connected to $v_{i}$, for $i \in\{0,1\}$. Assume that $H$ has no isolated vertices, and $S$ contains at least one neighbour of $v_{0}$, and one neighbour of $v_{1}$. 
This means that $a^{S}$ is a binary sequence such that $a^{S 0}$, and $a^{S 1}$ have at least one element which equals 0 . In this case $S$ is adjacent in $\gamma \cdot T$ to $S^{\prime}$ if $a^{S^{\prime} 0}=a^{S 0}$, and $a^{S^{\prime} 1} \neq(1,1 \ldots, 1)$ and differs from $a^{S 1}$ only at one coordinate, or symmetrically if $a^{S^{\prime} 1}=a^{S 1}$, and $a^{S^{\prime} 0} \neq(1,1 \ldots, 1)$ and differs from $a^{S 0}$ only at one coordinate. In this case $\gamma \cdot T \simeq Q_{n}^{-} \square Q_{m}^{-}, n+m=k$.

Let $H$ have at least one isolated vertex $v$. Assume that $v \in V\left(T_{0}\right)$. If there are no pendant vertices adjacent to $v_{1}$, then $|S|=k+l+1$. If there are more than two pendant vertices adjacent to $v_{0}$, then $v_{0} \in S$ dominates $v_{1}$. Furthermore, it is possible that $a^{S}$ is any binary sequence of length $k$, hence $\gamma \cdot T \simeq Q_{n}$. If $v$ is the only pendant vertex adjacent to $v_{0}$, then $\gamma \cdot T \simeq Q_{n}$ or $\gamma \cdot T \simeq Q_{n} \square Q_{m}^{-} \cdot \gamma \cdot T \simeq Q_{n}$ under the condition that $v_{1}$ is adjacent to a vertex $u$ of $T_{1}$ with degree greater than 2 . In this case $u$ belongs to every $\gamma$-set of $T$. In the other case $\gamma \cdot T \simeq Q_{n} \square Q_{m}^{-}$, because $S$ contains exactly one vertex from every $K_{1,1}$ component of $H$ and one vertex from the set $\left\{v, v_{0}\right\}$, and $a^{S 0}$ can be any binary sequence of an appropriate length, but $a^{S 1} * a_{k+1} \neq(1,1, \ldots, 1)$, where $a_{k+1}=1$ iff $v \in S$.

If also $v_{1}$ is adjacent to a pendant vertex, then $|S|=k+l+2$. Analogous reasoning lets us deduce that $\gamma \cdot T \simeq Q_{n}$.

Note that if $\gamma \cdot T \simeq Q_{n}^{-} \square Q_{m}$ or $\gamma \cdot T \simeq Q_{n}^{-} \square Q_{m}^{-}$, then $m+n \leq|V| / 2$. This proves the following fact.

COROLlary 2.5. If $T$ is a tree with $n$ vertices such that $\operatorname{diam}(T)=5$, then $\operatorname{diam}(\gamma \cdot T) \leq n / 2$.

\section{Gamma graphs of caterpillars with one leg}

In this section we consider certain trees with three pendant vertices. These trees can be obtained from a path by joining a new vertex to such vertex $v$ of this path which is not pendant. We will use the following notation. Let a caterpillar with one leg $\mathrm{Cat}_{n}^{s}$ be a graph defined in the following way: $V\left(\mathrm{Cat}_{n}^{s}\right)=V\left(P_{n}\right) \cup\{0\}$, and $E\left(\mathrm{Cat}_{n}^{s}\right)=E\left(P_{n}\right) \cup\{(0, s)\}$. For a caterpillar with one leg $G=\mathrm{Cat}_{n}^{s}$ let $G^{-}$denote the graph $\mathrm{Cat}_{n}^{s}-\{0, s\}$, and let $G^{--}=G^{-}-\{s-1, s+1\}$. Further we partition the class of caterpillars with one leg into the following six classes:

$$
\begin{aligned}
& \mathcal{C}_{! !}=\left\{\mathrm{Cat}_{3 k}^{s}: s \equiv 2(\bmod 3), 1<s<3 k, k \in \mathbb{N}\right\}, \\
& \mathcal{C}_{! 0}=\left\{\mathrm{Cat}_{3 k+2}^{s}: s \not \equiv 0(\bmod 3), 1<s<3 k+2, k \in \mathbb{N}\right\}, \\
& \mathcal{C}_{! 2}=\left\{\mathrm{Cat}_{3 k+1}^{s}: s \not \equiv 1(\bmod 3), 1<s<3 k+1, k \in \mathbb{N}\right\},
\end{aligned}
$$




$$
\begin{aligned}
& \mathcal{C}_{00}=\left\{\mathrm{Cat}_{3 k+1}^{s}: s \equiv 1(\bmod 3), 1<s<3 k+1, k \in \mathbb{N}\right\} \\
& \mathcal{C}_{02}=\left\{\mathrm{Cat}_{3 k}^{s}: s \not \equiv 2(\bmod 3), 1<s<3 k, k \in \mathbb{N}\right\} \\
& \mathcal{C}_{22}=\left\{\mathrm{Cat}_{3 k+2}^{s}: s \equiv 0(\bmod 3), 1<s<3 k+2, k \in \mathbb{N}\right\} .
\end{aligned}
$$

Note that $G^{-}$is a sum of two independent paths and that indices !, 0 and 2 indicate how many vertices belong to each component of $G^{-}$: the index ! corresponds to a component with $3 t+1$ vertices, 0 corresponds to a component with $3 t$ vertices, and 2 corresponds to a component with $3 t+2$ vertices. We use the index ! instead of 1 to stress that the component corresponding to this index can be omitted in finding $\gamma \cdot G$.

Corollary 3.1. If $v \in G$ is a pendant vertex adjacent to a cutvertex $c$ of $G-v$ and there exists a $\gamma$-set $S$ such that $v \in S$, then the subgraph $H$ of $\gamma \cdot G$ induced by $\gamma$-sets containing $v$ is a graph $H \simeq H_{1} \square H_{2} \square \ldots \square H_{k}$, where $H_{i}$ for $i \in\{1, \ldots, k\}$ are gamma graphs of components of $G-\{v, c\}$.

Proof. It suffices to prove that if $S$ is a $\gamma$-set of $G$ such that $v \in S$, then $S=\{v\} \cup S_{1} \cup S_{2} \cup \cdots \cup S_{k}$, where $S_{i}$ is a vertex of $H_{i}, i \in\{1,2, \ldots, k\}$. Note that $c \notin S$, otherwise $S \backslash\{v\} \subset S$ would be a dominating set such that $|S|<\gamma(G)$. Since $G-\{v, c\}$ is not connected and $c \notin S$, then $S$ contains a $\gamma$-set $S_{i}$ of every component of $G-\{v, c\}$. On the other hand every set $S$ such that $S=\{v\} \cup S_{1} \cup S_{2} \cup \cdots \cup S_{k}$, where $S_{1}, S_{2} \ldots S_{k}$ are gamma sets of components of $G-\{v, c\}$ dominates $G$.

Further we will use this corollary for caterpillars with one leg. Below we explicitly formulate the special case which is essential in proofs of final theorems.

Lemma 3.2. Let $H$ be a subgraph of $G=\gamma$. Cat $_{t}^{s}$ induced by all $\gamma$-sets of $G$ which contain 0 . Then $H=H_{1} \square H_{2}$, where vertices of $H_{1}$ and $H_{2}$ correspond to dominating sets of components of $G^{-}$.

A similar observation can be made for a subgraph $H$ of $G=\gamma$. Cat ${ }_{t}^{s}$ induced by all $\gamma$-sets of $G$ which contain $s$. Notice that $H=H_{1} \square H_{2}$, where vertices of $H_{1}$ and $H_{2}$ correspond to subsets of components of $G^{-}$which dominate components of $G^{--}$. These observations will be helpful in describing the structure of $\gamma$. $\mathrm{Cat}_{t}^{s}$.

TheOREM 3.3. Let $G=\gamma \cdot$ Cat $_{t}^{s}$, and let $H$ be a subgraph of $G$ induced by all $\gamma$-sets of $G$ which contain 0 .

If $G \in \mathcal{C}_{00}$, then $H \simeq K_{1}$,

if $G \in \mathcal{C}_{02}$, then $H \simeq P_{n}$,

if $G \in \mathcal{C}_{22}$, then $H \simeq P_{n} \square P_{m}$. 
It suffices to apply Lemma 3.2 to the appropriate caterpillar with one leg. The graph $H$ is a product of $\gamma \cdot G$ graphs of two paths.

Lemma 3.4. If $G \in \mathcal{C}_{! 0} \cup \mathcal{C}_{! 2} \cup \mathcal{C}_{! !}$, then the vertex 0 does not belong to any $\gamma$-set of $G$.

Proof. If $G=\mathrm{Cat}_{3 k+i}^{s} \in \mathcal{C}_{! 0} \cup \mathcal{C}_{! 2} \cup \mathcal{C}_{! !}$, then $P_{3 t+1}$ is a component of $G^{-}$. Suppose that $S$ is a $\gamma$-set such that $0 \in S$. Notice that $s \notin S$ because $S$ is minimal. It means that $|S|=1+(t+1)+(k-t)=k+2$. On the other hand if $S^{\prime}$ is a gamma set such that $0 \notin S^{\prime}$, then $s \in S^{\prime}$. No vertex of the graph $G^{--}$ is dominated by $s$, and domination numbers of its components are $\Gamma\left(P_{3 t}\right)=t$, and $\Gamma\left(P_{3(k-t-1)+i}\right) \leq k-t$, hence $\left|S^{\prime}\right| \leq 1+t+k-t=k+1$. Since $\left|S^{\prime}\right|<|S|$, then any gamma set of $\mathrm{Cat}_{3 k+i}^{s}$ cannot contain the vertex 0 .

Notice that if $S$ is a gamma set of $\mathrm{Cat}_{t}^{s}$ and $0 \in S$, then $S \backslash\{0\} \cup\{s\}$ is also a $\gamma$-set. Therefore if $H_{s}$ is a subgraph of $G$ induced by all $\gamma$-sets of $G$ which contain $s$ and $H_{0}$ is a subgraph of $G$ induced by all $\gamma$-sets of $G$ which contain 0 , then $H_{0}$ is isomorfic to an induced subgraph of $H_{s}$. This proves the following proposition.

Proposition 3.5. If $G=\mathrm{Cat}_{t}^{s}, H_{s}$ is a subgraph of $\gamma \cdot G$ induced by all $\gamma$-sets of $G$ which contain $s$ and $H_{0}$ is a subgraph of $\gamma \cdot G$ induced by all $\gamma$-sets of $G$ which contain 0 , then $V(\gamma \cdot G)=V\left(H_{s}\right) \cup V\left(H_{0}\right)$ and

$$
E(\gamma \cdot G)=E\left(H_{s}\right) \cup E\left(H_{0}\right) \cup\left\{(S, S \backslash\{0\} \cup\{s\}): S \in V\left(H_{0}\right)\right\} .
$$

Notice that for $\mathrm{Cat}_{t}^{s} \in \mathcal{C}_{! 0} \cup \mathcal{C}_{! 2} \cup \mathcal{C}_{\text {!! }}$ the graph $H_{0}$ is empty. In this case $\gamma$. Cat $_{t}^{s} \simeq H_{s}$. In order to complete the description of $\gamma$. Cat $_{t}^{s}$ graphs it suffices to prove the following theorem.

TheOREm 3.6. Let $G=\gamma$. Cat $t_{t}^{s}$. If $H$ is a subgraph of $G$ induced by all $\gamma$-sets of $G$ which contain $s$, then there exist $n, m \in \mathbb{N}$ that:

if $G \in \mathcal{C}_{00}$, then $H \simeq P_{n} \square P_{m}$,

if $G \in \mathcal{C}_{02}$, then $H \simeq P_{n} \square S l(n)$,

if $G \in \mathcal{C}_{22}$, then $H \simeq S l(n) \square S l(m)$,

if $G \in \mathcal{C}_{! 0}$, then $H \simeq P_{n}$,

if $G \in \mathcal{C}_{! 2}$, then $H \simeq S l(n)$,

if $G \in \mathcal{C}_{\text {!! }}$, then $H \simeq K_{1}$.

Proof. Let $S$ be a $\gamma$-set of $G$. If $G \in \mathcal{C}_{! 0} \cup \mathcal{C}_{! 2} \cup \mathcal{C}_{! !}$, then $P_{3 n+1}$ is a component of $G^{-}$. We can assume that $n>1$, and then $P_{3 n}$ is one of the components of $G^{--}$. Additionally, $n$ vertices of $S$ which dominate this component belong to its $\gamma$-set. Otherwise one of the pendant vertices of $P_{3 n}$ would be dominated 
by a vertex of $G^{-}-G^{--}$, but the vertices of $P_{3 n-1}$ cannot be dominated by a set of $n-1$ vertices. In this case $S=\Gamma \cup\{s\} \cup \Gamma^{\prime}$, where $\Gamma$ is the only $\gamma$-set of $P_{3 n}$, and $\Gamma^{\prime} \subset V\left(G^{-}-P_{3 n+1}\right)$ dominates vertices of the other component of $G^{--}$.

If $G \in \mathcal{C}_{! 0} \cup \mathcal{C}_{00} \cup \mathcal{C}_{02}$, then $P_{3 n}$ is a component of $G^{-}$, and then $P_{3(n-1)+2}$ is one of the components of $G^{--}$. Similarly, like in the previous case, $n$ vertices of $S$ which dominate this component belong to its $\gamma$-set. Hence $S=\Gamma \cup\{s\} \cup \Gamma^{\prime}$, where $\Gamma$ is a $\gamma$-set of $P_{3(n-1)+2}$, and $\Gamma^{\prime} \subset V\left(G^{-}-P_{3 n+1}\right)$ dominates the other component of $G^{--}$. In other words, $\Gamma$ corresponds to a vertex of the graph $\gamma \cdot P_{3(n-1)+2} \simeq P_{n-1+2}$.

If $G \in \mathcal{C}_{! 2} \cup \mathcal{C}_{02} \cup \mathcal{C}_{22}$, then $P_{3 n+2}$ is a component of $G^{-}$and $P_{3 n+1}$ is one of the components of $G^{--}$. We can assume that $V\left(P_{3 n+2}\right)=\{1,2, \ldots, 3 n+2\}$ and $3 n+3=s$. In this case $n+1$ vertices of $S$ which dominate $P_{3 n+1}$ either belong to its $\gamma$-set or $3 n+2$ is one of these vertices and $n$ other vertices dominate the path $P_{3 n}$, to which they belong. Assume that the second case is true and notice that the $n$ vertices which dominate $P_{3 n}$ is the unique $\gamma$-set $S_{0}$ of this path, where $S_{0}=\{3 i+2: 0 \leq i<n\}$. Hence $S=\Gamma \cup\{s\} \cup \Gamma^{\prime}$, where $\Gamma$ is a $\gamma$-set of $P_{3 n+1}$ or $\Gamma=S_{0} \cup\{3 n+2\}$, and $\Gamma^{\prime} \subset G^{-}-P_{3 n+2}$ dominates the other component of $G^{--} . S_{0} \cup\{3 n+1\}$ and $S_{0} \cup\{3 n\}$ are the only $\gamma$-sets of $P_{3 n+1}$ which have only one element different from the set $S_{0} \cup\{3 n+2\}$. This proves that subsets of $\gamma$-sets of $G$ which dominate the component $P_{3 n+1}$ correspond to vertices of the graph obtained from $S G^{+}(n)$ by adding one new vertex and two edges. This graph is isomorfic to $\operatorname{Sl}(n)$.

If $G \in \mathcal{C}_{! 0} \cup \mathcal{C}_{! !} \cup \mathcal{C}_{! 2}$, then one component of the graph $G^{-}$is a path $P_{3 t+1}$. If additionally $t=0$, then $G^{--}$has only one component $P_{3(k-1)+i}, i \in\{0,1,2\}$.

If $G^{--}$has exactly two components $G_{1}$ and $G_{2}$, then every $\gamma$-set of $G$ which contains $s$ is a set $\{s\} \cup \Gamma_{1} \cup \Gamma_{2}$, where $\Gamma_{1}$ dominates $G_{1}$ and $\Gamma_{2}$ dominates $G_{2}$. Two such gamma sets $\{s\} \cup \Gamma_{1} \cup \Gamma_{2}$ and $\{s\} \cup \Gamma_{1}^{\prime} \cup \Gamma_{2}^{\prime}$ are adjacent iff $\Gamma_{1}=\Gamma_{1}^{\prime}$ and $\Gamma_{2}$ has only one element different from $\Gamma_{2}^{\prime}$, or $\Gamma_{1}$ has exactly one element different from $\Gamma_{1}^{\prime}$ and $\Gamma_{2}=\Gamma_{2}^{\prime}$. Hence $\gamma \cdot G \cong H_{1} \square H_{2}$, where $H_{1}$ and $H_{2}$ are isomorfic to a path, slide or a graph $K_{1}$.

We finish with observations on the size of diameter of $\gamma$. Cat $t_{t}^{s}$. Let $H_{0}$ and $H_{s}$ be induced subgraphs of $\gamma$. $\mathrm{Cat}_{t}^{s}$ defined as in Proposition 3.5. It is obvious that $\operatorname{diam}\left(H_{0}\right)<\operatorname{diam}\left(H_{s}\right)$. Since every vertex of $H_{0}$ is adjacent in $\gamma$. Cat $t_{t}^{s}$ to some vertex of $H_{s}$, we immediately conclude that $\operatorname{diam}\left(\gamma \cdot \mathrm{Cat}_{t}^{s}\right) \leq \operatorname{diam}\left(H_{s}\right)+$ 1. Furthermore it is easy to show that $\operatorname{diam}(S l(n))=n, \operatorname{diam}\left(G_{1} \square G_{2}\right)=$ $\operatorname{diam}\left(G_{1}\right)+\operatorname{diam}\left(G_{2}\right)$. The proof of the theorem shows that the ratio between $\left|V\left(H_{s}\right)\right|$ and $\left|V\left(\mathrm{Cat}_{t}^{s}\right)\right|$ is the biggest when $\mathrm{Cat}_{t}^{s} \in \mathcal{C}_{00}$. In this case $H_{s}$ is a product of two paths and for every $\gamma$-set $S=\Gamma_{1} \cup\{s\} \cup \Gamma_{2} \in V\left(H_{s}\right)$ the sets $\Gamma_{1}$ and $\Gamma_{2}$ correspond to some vertices of $\gamma \cdot P_{3 k+2} \simeq P_{k+2}$ and $\gamma \cdot P_{3 l+2} \simeq$ $P_{l+2}$, where $\mid V\left(\right.$ Cat $\left._{t}^{s}\right) \mid=(3 k+2)+(3 l+2)+3+1=3(k+l)+8$. Then 
$\left|V\left(G_{1}\right)\right|+\left|V\left(G_{2}\right)\right|=l+k+4$ and hence $\operatorname{diam}\left(H_{s}\right)=(n+4) / 3$. Therefore, the following proposition holds.

Proposition 3.7. If $G=\mathrm{Cat}_{t}^{s}$ is a caterpillar with one leg then

$$
\operatorname{diam}(\gamma \cdot G) \leq(t+5) / 3+1
$$

\section{References}

[1] Diestel R., Graph theory, Springer-Verlag, Heidelberg, 2005.

[2] Fricke G.H., Hedetniemi S.M., Hedetniemi S.T., Hutson K.R., $\gamma$-graphs of graphs, Discuss. Math. Graph Theory 31 (2011), 517-531.

[3] Haas R., Seyffarth K., The k-dominating graph, Graphs Combin. 30 (2014), 609-617.

[4] Haynes T.W., Hedetniemi S.T., Slater P.J., Fundamentals on domination in graphs, CRC Press, New York, 1998.

[5] Lakshmanan S.A., Vijayakumar A., The gamma graph of a graph, AKCE J. Graphs Combin. 7 (2010), 53-59.

Institute of Mathematics

University of Silesia

BANKOWA 14

40-007 Katowice

POLAND

e-mail: anna.bien@us.edu.pl 